\title{
POWER GENERATION BY UTILIZING REPULSIONS OF PERMANENT MAGNETS FOR SMALL SCALE APPLICATIONS
}

\author{
Ismail Khan ${ }^{1}$, Chethan K M², Avinash $\mathbf{H}^{\mathbf{3}}$, Prakash $\mathrm{T}^{\mathbf{4}}$

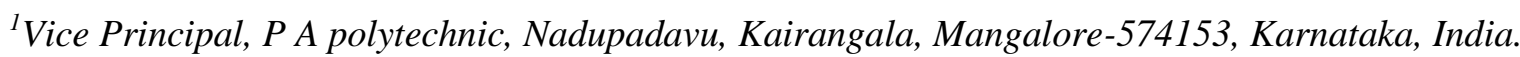 \\ ${ }^{2}$ Assistant Professor, Department of Mechanical Engineering, PACE, Mangalore-574153, Karnataka, India \\ ${ }^{3}$ Assistant Professor, Department of Automobile Engineering, SIT, Mangalore, 574143, Karnataka, India. \\ ${ }^{4}$ Assistant Professor, Department of Automobile Engineering, SIT, Mangalore, 574143, Karnataka, India.
}

\begin{abstract}
We are generating energy from various sources like wind energy, solar energy, geo thermal energy ocean thermal energy etc., which is naturally available in free of cost. But all this sources have some limitation where we cannot depend on solar energy During rainy season, wind energy during summer season etc., due to scarcity of power generation much modern technique must be developed to generate energy in the modern world hence the concept is to generate the energy using magnets as the sources, This process helps to generate energy during required time without depending on naturally available resources. In this project we are making use of permanent magnet which produces magnetic field which is invisible but it is responsible for notable property, when two magnets of different poles bought together they develop repulsion forces and due this repulsion forces we are making shaft to rotate which will be connected to the DC generator to produce electricity.
\end{abstract}

Keywords: Scarcity, Technique, Repulsion, notable, Generator,

\section{INTRODUCTION}

The repulsive type magnetic system has been used so far in various kinds of bearing. Due to the shortage of power generation, much new latest technique should be encouraged and developed for electricity production in this world. This made us to think and invent generating electricity from repulsion which will be caused in permanent magnet. The technique is very effective and less in cost. This generated power can be utilized in all kind of applications, the generated power can be send to UPS. Where UPS is used in during power shut down. This type of system can be used in critical situations such as on-line system for banking, life-supporting system like during operation theaters etc. Therefore it is important that this system is designed to be smaller and efficient, this type of power generation techniques can be used in remote areas where still some villages around the world have no electricity due to lack of facilities. Hence this type of power generation method can be adopted in these areas.

The system which we are developing uses permanent magnets as the main object to develope repulsion and this repulsive force creates a torque which will be transmitted to the shaft that drives a DC generator. The repulsive magnetic system consists of two main magnetic discs of which one is mounted in the rotating machine and the second one is connected to the DC generator. The repulsion between the two magnets makes the second disc to rotate which develops the primary input for DC generator. Thus the energy is available in free of cost by the technique which we have developed. The output power which is obtained from the DC generator can be stored in batteries or directly it can be used to run electric appliances.

\section{LITERATURE SURVEY}

The concept Magnetism which can be called as physical quantity that is obtained only by magnetic fields. Electric currents also the moments of magnets and their particles makes the magnetic field, which acts as opposite current to develop repulsion which can also be called as movements of magnet . The magnetic moments or repulsion will be more in ferromagnetic materials, which is strongly attracted by magnetic fields and this is magnetized and made permanent magnets. Every magnet has two points, which is named as poles, where the maximum magnetic strength is concentrated, they are called as North Pole, and South Pole, since all magnets will be having two poles, and it can be called as magnetic dipoles. The most important property of these poles are that like poles always repels to each other, and the unlike poles get attracted to each other.

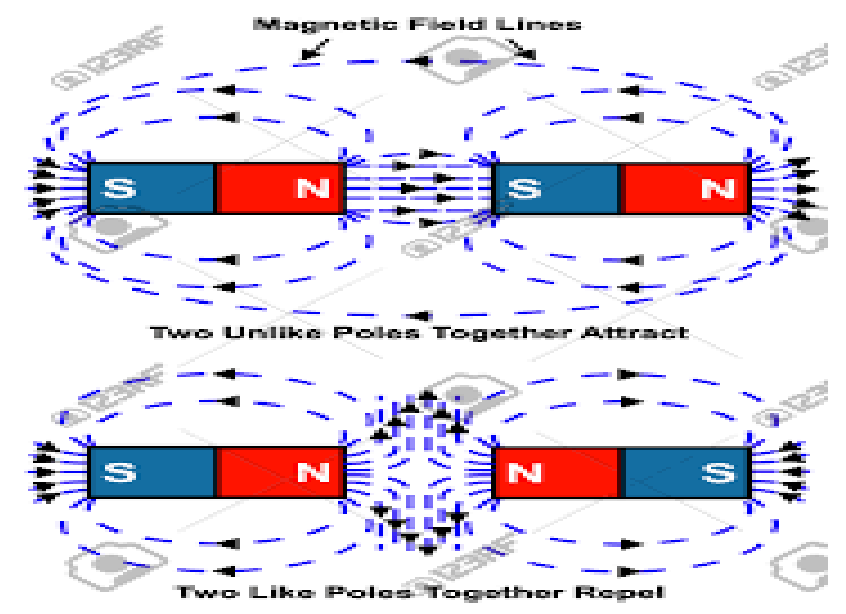

Fig 1: Attraction and repulsion of magnets 
Many scientists have proved that the magnetic fields which will are produced by permanent magnets will pushes or try to pull the electrons of some metal objects like iron copper etc., if we are making permanent magnet to move fastely through copper wire coils the electrons are also made move and electricity can be generated, Also many power plants are making use of the magnets to convert the kinetic energy developed in permanent magnet into useful electric energy.

Hans Christian in 1819 during his teachings accidently came to know that magnets can also generate electricity when they are allowed to move around coils of copper wires, so later on with is continuous effort and practice of certain experiment on magnets he was able to create electric field using magnets as the main sources. During our literature survey, maglev train which is the fastest train in the world which uses magnet repulsion system to move the train, they uses the same repulsion principle in such a way that the arrangements of magnets which they named as magnetic river that will make the linear motor to create lift and forward thrust which makes the above object to flow through it. Hence after collecting the information from various sources we decided to design our own system.

\section{OBJECTIVES OF THE WORK}

The main objective of our project is to develop the technique to rotate a shaft without using any external sources like solar energy wind energy, water energy etc., thus by using permanent magnet as the source and making use of its property we are driving a shaft with the help of externally arranged semicircled pulleys which can rotate due to repulsive action of the permanent magnets and these shaft are connected to the generator to produce electricity.

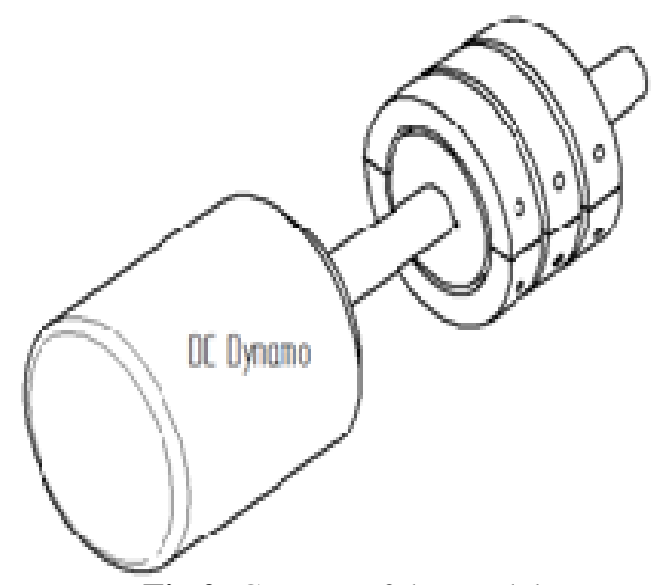

Fig 2: Concept of the Model

\section{METHODOLOGY}

We are making use of permanent magnet and magnetic repulsion is caused by centrifugal force acting between outer pulley arrangements and the permanent magnets around the shaft, arrangements and methods are simple, the DC dynamo are mounted on the platform where the shaft is connected to the dynamo and the semicircle pulley with permanent magnets are fixed around the shaft at certain distance, initially some external force is required to rotate the shaft and once the twisting movement is generated in the axis of rotation the dynamo starts rotating hence electricity is produced.

We have made use of 3D CAD modeling software (Solid Edge) to design the concept. Solid Edge 3D CAD modeling mechanical software is easy to do part sketching, part design and assemble the part design and also some of the design and data-management solutions which include applications for PDM, analysis, and CAM. Solid Edge will be useful for both designers and engineers.

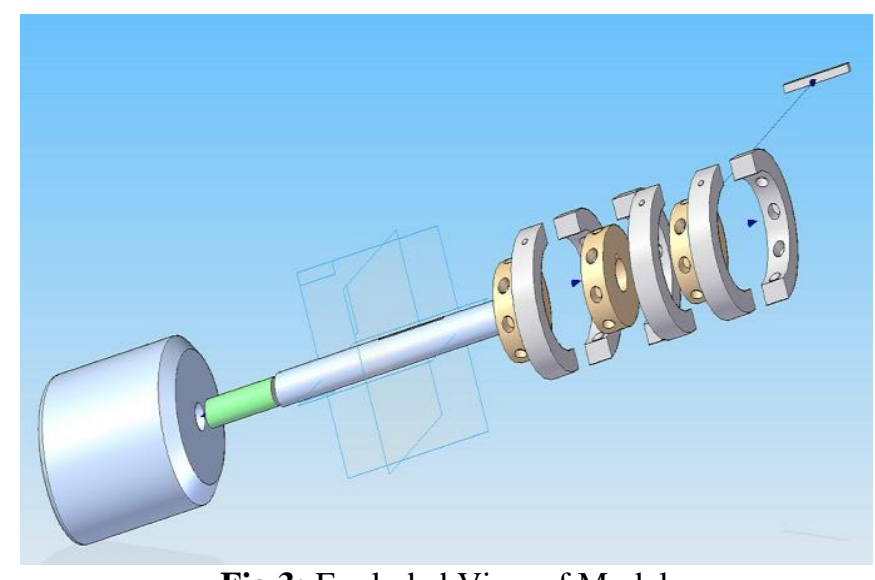

Fig 3: Exploded View of Model

The power generating by magnetic repulsion converts this kinetic energy into electrical energy. The magnets are fitted on the outer side of the pulleys and for a cap on the inner side of the periphery. The first initial rotation given to shaft and pulley then suddenly close the outer cap and fix the clamps, then automatically shaft starts rotating due to its magnetic effect.

The magnets repels to each other on the both side of the pulley and then the inertia force on the pulley that makes to rotate a shaft which rotates about 200-600 rpm, the other end of the shaft is connected to DC Dynamo, this converts the rotational energy into electrical energy. It gets output around 12.81 volts for $240 \mathrm{rpm}$. Based on our trial run.

\section{COMPONENTS USED IN PROJECT}

\subsection{Dynamo}

The electric dynamo uses rotating coils of copper wires and magnetic fields to convert mechanical rotation energy into electric current. A dynamo machine consists of a semi stationary structure, named as stator, which creates a constant magnetic field, and a set of rotating windings called as armature which rotates within that filed, the moment of inertia of copper wire within the magnetic field creates an electromotive force that pushes the electrons of the metal, in small machines the constant magnetic field can be generated by one or more permanent magnets, larger machines have the constant magnetic field provided by one or more electromagnets, which are called as field coils. 


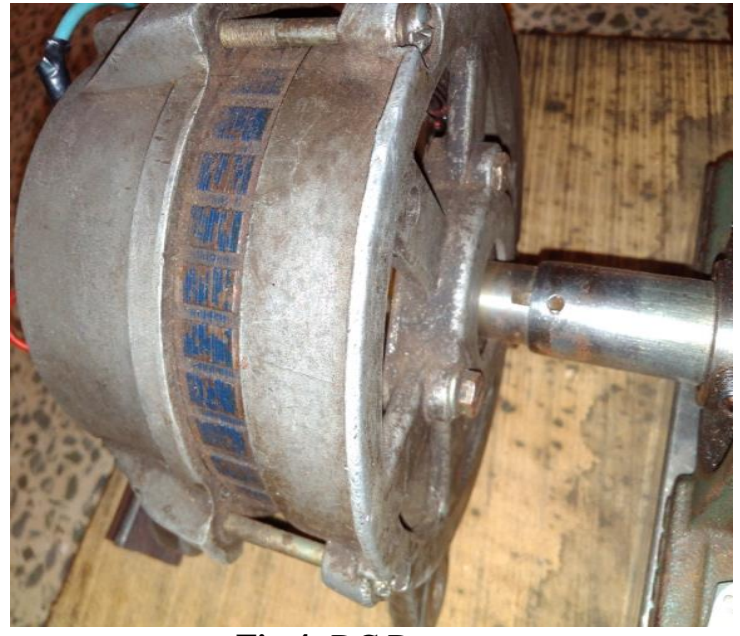

Fig 4: DC Dynamo

\subsection{Shaft}

A shaft is other important component which rotate machine element to transmit power from one part to another, they are mainly classified into two types.

a. Transmission shafts are used to transmit power between the source and the machine absorbing power; e.g. counter shafts and

b. Machine shafts which are the integral part of the Materials.

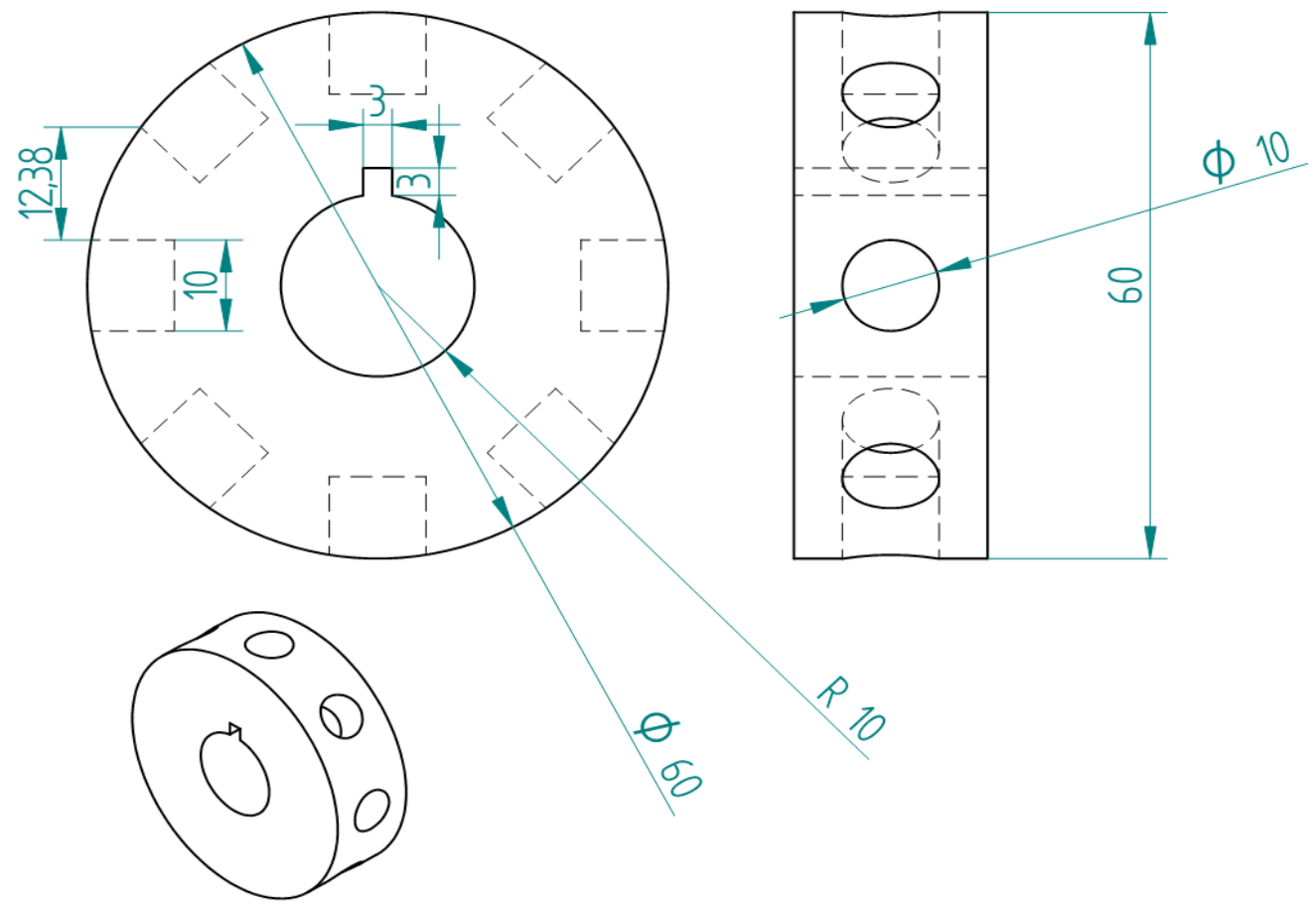

We have used mild steel as shaft material of diameter $20 \mathrm{~mm}$ and $203 \mathrm{~mm}$ length.

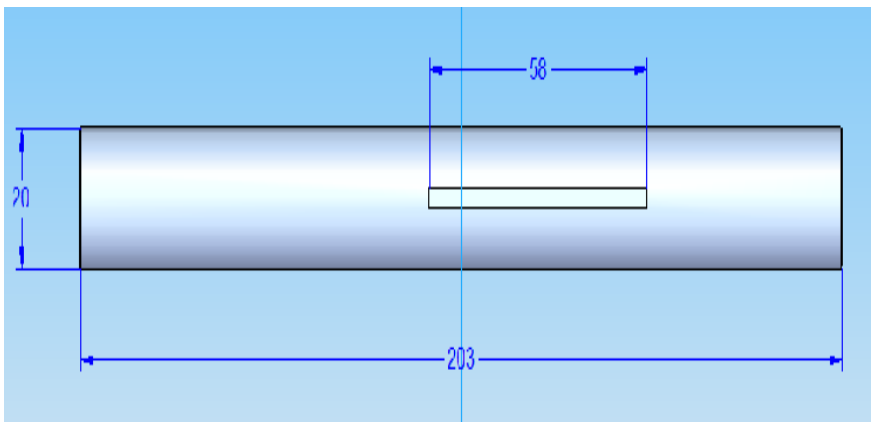

Fig 5: Shaft

\subsection{Pulley}

It is made up of aluminium, the weight of the pulley is $109 \mathrm{gm}$ and it has 8 hole of $10 \mathrm{~mm}$ diameter on the outer periphery of the pulley.

Fig 6: Pulley

\subsection{Outer Cap of Pulley}

It is made up of aluminium same as pulley and it contains 9 holes of $10 \mathrm{~mm}$ diameter on inner periphery of the cap. Specification of the outer cap as shown in below figure, 

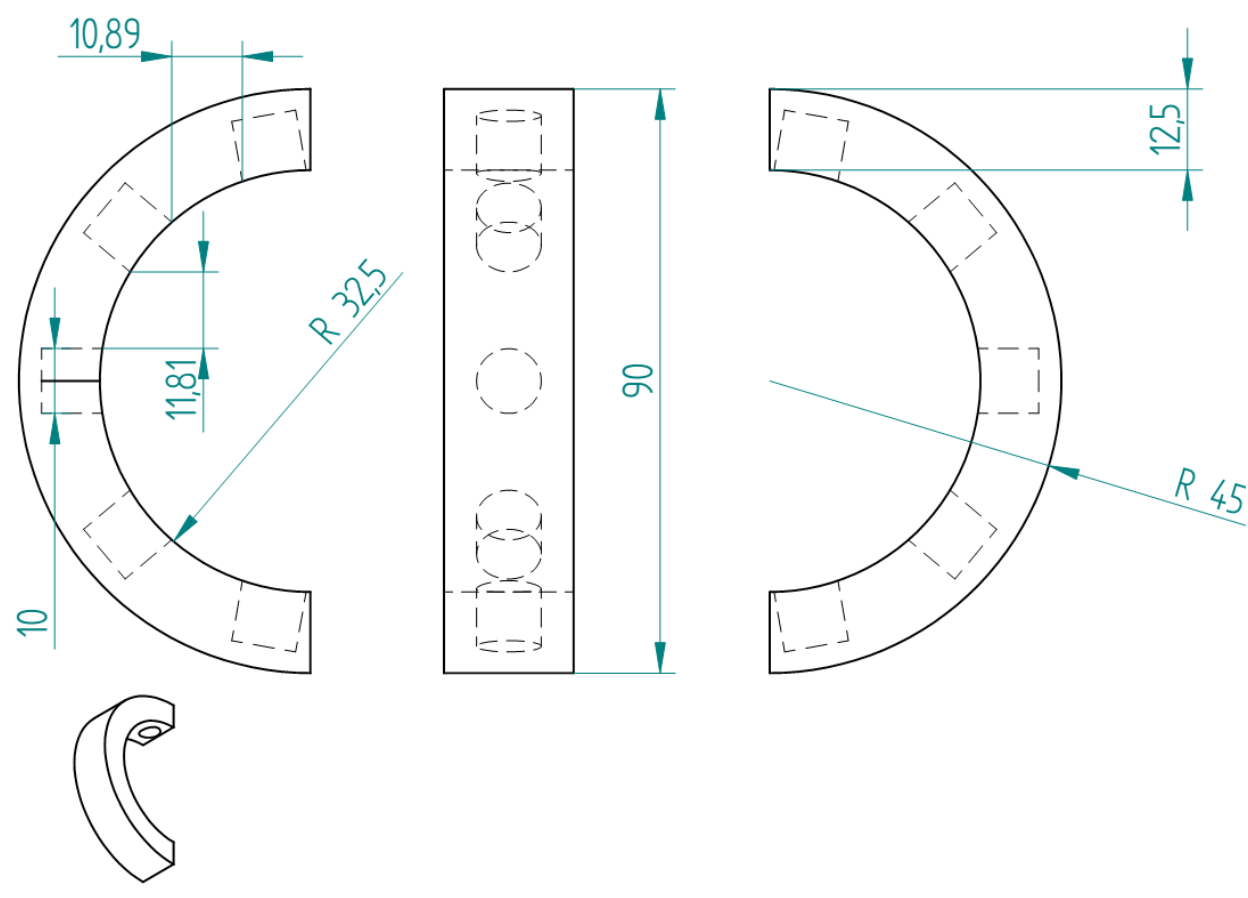

Fig 7: Outer Cap of Pulley

\subsection{Plummer Block with Ball Bearing}

A Plummer block which is also known as a pillow block or housed bearing unit, it is a pedestal used to provide support for a rotating shaft with the help of compatible bearings \& various accessories. Housing material for a pillow block is specially made up of cast iron or cast steel. In this project we have used Plummer of internal diameter $20 \mathrm{~mm}$, external diameter $45 \mathrm{~mm}$, length of the Plummer is $125 \mathrm{~mm}$ and width is $35 \mathrm{~mm}$.

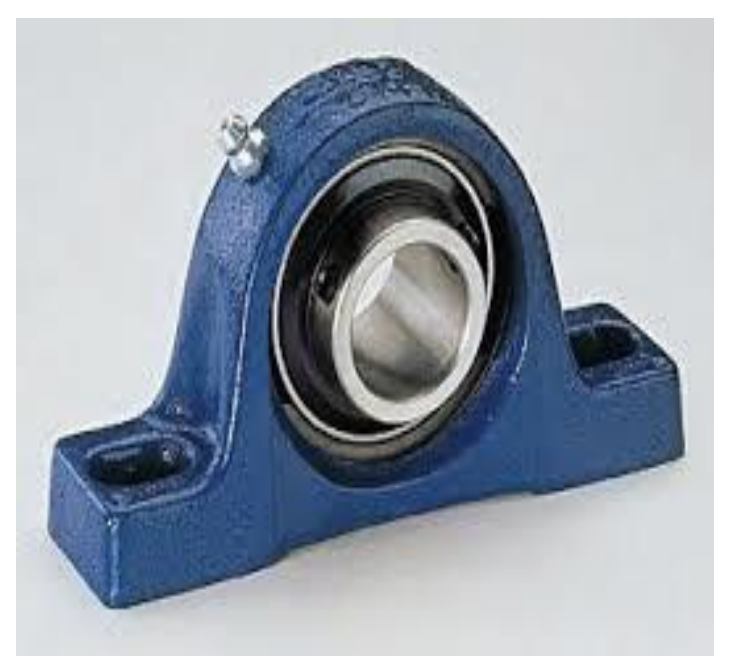

Fig 8: Plummer Block with Ball Bearing

\subsection{Neodymium Magnet}

It is the most common and widely used type of rare-earth magnet, is a permanent magnet made from an alloy of neodymium, iron and boron to form the $\mathrm{Nd} 2 \mathrm{Fe} 14 \mathrm{~B}$ tetragonal crystalline structure.

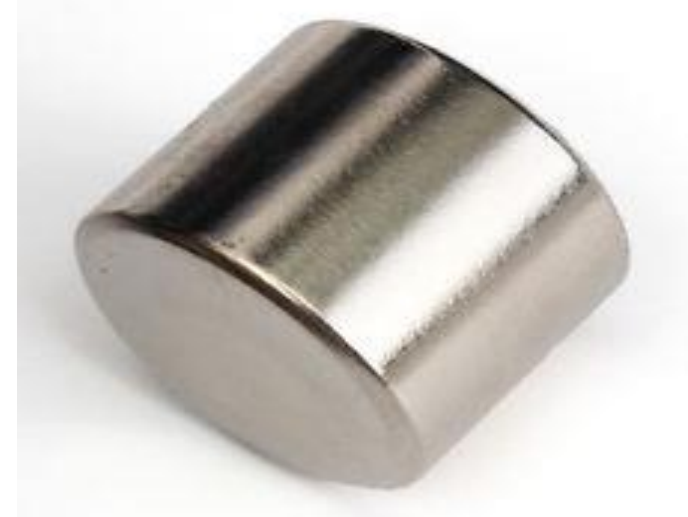

Fig 9: Neodymium Magnet

\subsection{Hinges, Nuts, Bolts and Screw}

A hinge is a mechanical bearing that connects two solid objects, typically allowing only a limited angle of rotation between them. Two objects connected by an ideal hinge rotate relative to each other about a fixed axis of rotation.

A nut is a type of fastener with a threaded hole. Nuts are always used in conjunction with a mating bolt to fasten multiple parts together.

Bolt is a form of threaded fastener with an external male thread. A screw is a type of fastener, sometimes similar to a bolt, which is made of metal, and characterized by a helical ridge, known as a male or thread external thread or just thread.

And screw is an inclined plane which is wrapped around a nail 


\section{CONCLUSION}

This principle uses permanent magnet for developing repulsive force where the shaft is connected to dynamo and dynamo on the platform we are using semicircle pulleys mounted on the shaft at certain distance where permanent magnet are placed in between the pulleys repulsion of magnets takes place and shaft rotates this rotational energy of shaft converted into electrical energy using DC Dynamo

In this project an attempt is made to bring out an easier and cost efficient way of generating power for small scale application. During testing our device, at $240 \mathrm{rpm}$ of shaft speed, DC Dynamo output was 12.81 volts and torque generated is 14.83 $\mathrm{N}-\mathrm{m}$.

At 230 rpm of shaft speed, DC Dynamo output was 11.32 volts and torque generated is $15.89 \mathrm{~N}-\mathrm{m}$.

This project can be built in large model to produce large amount of power in future.

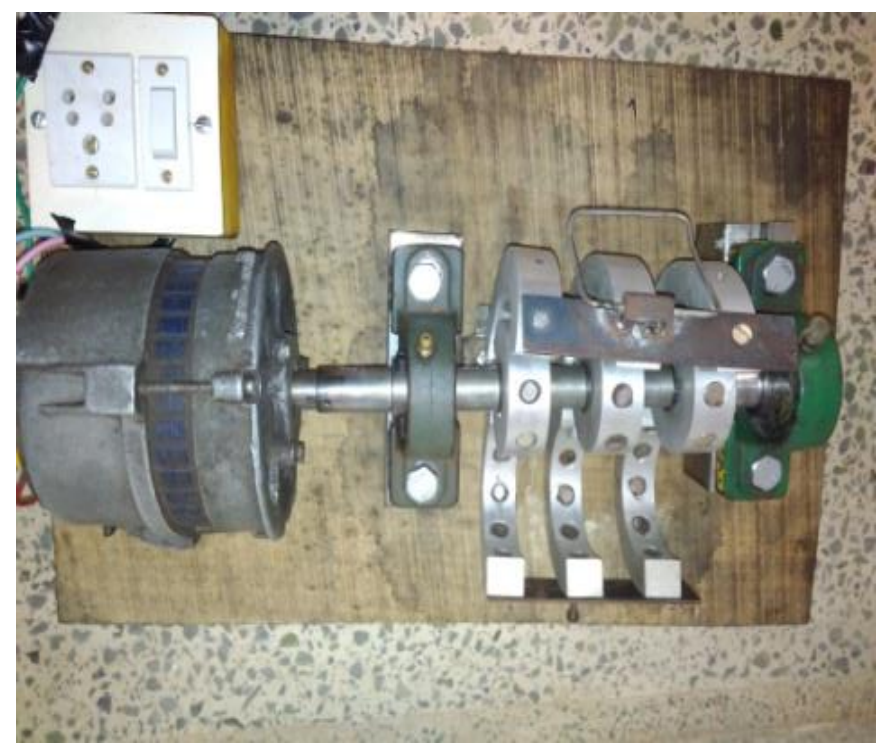

Fig 10: Assembly and Working of Model

\section{REFERENCES}

[1] Bose BK. Power Electronics and Motor Drives Advances and Trends. San Diego, CA, USA: Academic Press, 2006.

[2] Clerk-Maxwell, J., "On Physical Lines of Force", Philosophical Magazine, Volume 21, (1861)

[3] Eclipse Magnetics, "A world leader in magnetic technology"

[4] Frederick David Tombe, "Magnetic Repulsion and Centrifugal Force", Published $10^{\text {th }}$ April 2010.

[5] Subrahmanyam V. Electric Drives Concepts and Applications. Second eddision. New Delhi, India: Tata McGraw-Hill, 2011.

[6] T.Meenakshi, S.Sujithkumar, S.Suganya, Electricity Generation By Employing Repulsion Magnetic Technique In Rotating Machines, International Conference on Advances in Engineering \& Technology - 2014 (ICAET-2014), PP 47-52
[7] Suthanthira Vanitha Narayanan, Meenakshi, Sujithkumar Sivaraman, "Certain investigations on power generation using repulsive magnets and new stepped DC coupled quasi Z-inverter", Turkish Journal of Electrical Engineering \& Computer Sciences (2016).

\section{BIOGRAPHIES}

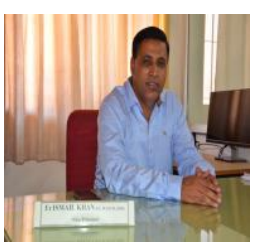

Ismail Khan BE, M-Tech, (phd), Vice Principal, P A POLYTECHNIC, Dept of Mechanical Engineering, Nadupadavu, kairangala, Mangalore-574153

Karnataka. India.

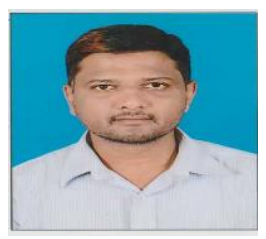

Chethan K M, Assistant Professor, Dept of Mechanical Engineering, P A College of Engineering, Mangalore-574153, Karnataka, India.

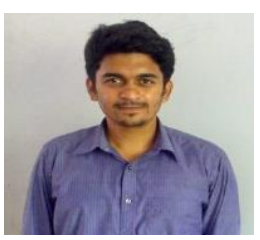

Avinash H S, Assistant Professor, Dept of Automobile Engineering, Srinivasa Institute of Technology, Mangalore, 574143, Karnataka, India.

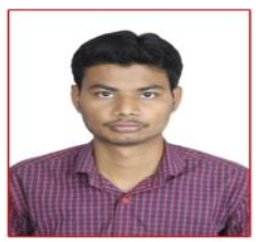

PRAKASH T, Assistant Professor, Dept of Automobile Engineering, Srinivasa Institute of Technology, Mangalore, 574143, Karnataka, India. 